\title{
CFTR mutation enhances Dishevelled degradation and results in impairment of Wnt-dependent hematopoiesis
}

\author{
Huaqin Sun', Yan Wang², Jieting Zhang², Yan Chen', Yanyan Liư ${ }^{3}$ Ziyuan Lin', Mingfeng Liu', Kai Sheng', \\ Huijuan Liao', Kam Sze Tsang ${ }^{4}$, Xiaohu Zhang ${ }^{1}$, Xiaohua Jiang ${ }^{2}$, Wenming X ${ }^{1}$, Meng Mao ${ }^{1}$ and Hsiao Chang Chan ${ }^{1,2}$
}

\begin{abstract}
Mutations of cystic fibrosis transmembrane conductance regulator (CFTR) cause cystic fibrosis (CF) with a multitude of clinical manifestations. Some CF patients develop clinically significant anemia, suggesting that CFTR may regulate hematopoiesis. Here, we report that cftr mutant zebrafish model exhibits primitive and definitive hematopoietic defects with impaired Wnt signaling. Cftr is found to interact, via its PDZ-binding domain (PDZBD), with Dishevelled (Dvl), a key component of Wnt signaling required for hematopoietic progenitor specification, thus protecting Dvl from Dapper1 (Dpr1)-induced lysosomal degradation. Defective hematopoiesis and impaired Wht signaling in cftr mutant can be rescued by overexpression of wild-type or channel function-defective G551D mutant CFTR with an intact PDZBD, but not Cftr with mutations in the PDZBD. Analysis of human database (http://r2.amc.nl) shows that CFTR is positively correlated with DVL2 and Wnt-related hematopoietic factors in human blood system. The results reveal a previously unrecognized role of CFTR, which is independent of its channel function, in regulating DVL degradation and thus Wnt signaling required for hematopoiesis in both zebrafish and humans, providing an explanation for the anemic phenotype of CF patients.
\end{abstract}

\section{Introduction}

Cystic fibrosis transmembrane conductance regulator (CFTR) is an ATP-binding cassette (ABC) transporter superfamily cAMP-activated anion channel ${ }^{1}$. It was first found to be expressed in a wide variety of epithelial tissues $^{2}$. Mutations of the CFTR have been shown to cause cystic fibrosis (CF), the most common lethal genetic disease in Caucasians with a hallmark defect in electrolyte and fluid transport affecting multiple organ systems with

\footnotetext{
Correspondence: Meng Mao (dffmmao@126.com) or Hsiao Chang. Chan (hsiaocchan@cuhk.edu.hk)

${ }^{1}$ SCU-CUHK Joint Laboratory for Reproductive Medicine, Key Laboratory of Birth Defects and Related Diseases of Women and Children (Sichuan University), Ministry of Education, Department of Pediatrics, West China Second University Hospital, Sichuan University, 610041 Chengdu, China

${ }^{2}$ Epithelial Cell Biology Research Center, School of Biomedical Sciences, Faculty of Medicine, The Chinese University of Hong Kong, Hong Kong SAR, China

Full list of author information is available at the end of the article

Edited by $\mathrm{H}-\mathrm{U}$ Simon
}

a multitude of clinical manifestations ${ }^{3,4}$. Defective CFTR ion channel function due to the most common mutation of CFTR, $\triangle$ F508 mutation, has been shown to underline the pathogenesis of some of the disease conditions in CF, such as obstructive lung disease $e^{5,6}$, pancreas exocrine deficiency $^{7}$, CF-related diabetes ${ }^{8}$, abnormal gonad function, and infertility ${ }^{9-11}$. However, many CF symptoms cannot be explained by a disease mechanism based on channelopathy.

Accumulating evidence has indicated that, in addition to its channel function, CFTR may also act as a potential regulator via its interaction with a large number of proteins $^{12}$. Notably, CFTR has a PDZ-binding domain (PDZBD) at its carboxy terminus, which can bind to proteins with PDZ domain ${ }^{13}$, the most abundant protein interaction module in the human genome ${ }^{14}$. Our previous study has found that CFTR interacts with adherens junction molecule AF-6/afadin via PDZBD, thus 
regulating epithelial polarity and affecting cancer metas$\operatorname{tasis}^{15}$. Ruan et al. have recently demonstrated that CFTR interacts with ZO-1 through PDZBD, and modulates the expression of ZO-1-ZONAB pathway downstream genes in cell proliferation and differentiation ${ }^{16}$. Of note, this effect was observed in the absence of cAMP stimulation, a condition that keeps CFTR channel open probability near zero, suggesting that the channel activity of CFTR is not required for its role in tight junction assembly. However, whether CFTR channel activity is required for its regulatory role has not been rigorously tested. Nevertheless, the demonstrated ability of CFTR to interact with an array of proteins and regulate different cellular processes has shed new light on the pathogenesis of myriad clinical manifestations of CF, which cannot be explained simply by a defect in CFTR channel function. For example, severe anemia in CF has also been reported in $4 \%$ of CF infants ${ }^{17}$. This suggests possible and yet unexplored involvement of CFTR in hematopoiesis.

Wnt/ $\beta$-catenin signaling plays a major role in hematopoiesis $^{18}$. Dishevelled (Dvl) is a crucial adaptor protein, which also contains a PDZ domain, in the canonical Wnt signaling pathway that leads to the nuclear translocation of $\beta$-catenin. Wnt signaling ultimately activates the $\mathrm{Cdx}$ Hox pathway, which drives the expression of key transcription factors, such as $s c l$ and gatala, that are central to the specification of hematopoietic precursors ${ }^{19,20}$.

Controlling Dvl protein stability is an important mechanism for the cells to regulate the Wnt signaling pathway. Dvl is degraded through either the proteasomal or lysosomal pathway ${ }^{21}$. Several proteins have been reported to induce Dvl proteasomal degradation ${ }^{21-23}$, whereas the study on Dvl lysosomal degradation is less extensive. Dapper1 (DPR1) has been identified as a Dvlinteracting protein that promotes Dvl turnover through lysosomes $^{24}$. Specifically, DPR1 enhances the interaction of Dvl2 with the Von Hippel-Lindau tumor suppressor (pVHL), an E3 ubiquitin ligase component, resulting in Dvl2 ubiquitination and degradation in lysosomes ${ }^{25}$.

The zebrafish has emerged to be an ideal model organism for hematopoiesis research owing to a number of experimental advantages, including short lifespan, external development, transparent embryos, and genetic amenability $^{26}$. Similar to other vertebrate organisms, zebrafish also have two waves of hematopoiesis, which occur in a spatially unique manner ${ }^{27}$. Zebrafish primitive hematopoiesis takes place in ventrolateral plate mesoderm-derived tissue called intermediate cell mass (ICM), which gives rise to erythrocytes in the posterior part of the embryo and generates myeloid cells in the anterior part of the embryo ${ }^{28-30}$. After primitive hematopoiesis, definitive hematopoietic stem cells (HSCs) emerge from the ventral wall of dorsal aorta in a region known as aorta-gonad-mesonephros $(\mathrm{AGM})^{30}$, and migrate to the posterior region in the tail called the caudal hematopoietic tissue $(\mathrm{CHT})$, before seeding the kidney marrow, which is equivalent to bone marrow in mammals, where HSCs produce blood cells of all lineages for the rest of the lifespan ${ }^{28,29}$. Importantly, the programs controlling hematopoiesis in zebrafish, including Wnt signaling, are conserved in mammals including humans, making zebrafish a clinically relevant model system ${ }^{26}$.

Given the clinical incidences of anemia in CF patients and the importance of Wnt signaling in hematopoiesis, we hypothesized that CFTR might regulate Wnt-dependent hematopoiesis through a potential interaction between Cftr and Dvl via their PDZBD/PDZ domain. We undertook the present study to test this hypothesis using zebrafish model, and revealed a previously unrecognized role of CFTR, which is independent of its channel function, in the Wnt signaling pathway crucial for both primitive and definitive hematopoiesis, providing an explanation as to how CFTR mutation may lead to anemia in CF patients.

\section{Results}

cftr mutant leads to primitive and definitive hematopoietic deficiency in zebrafish

Zebrafish Cftr, similar to human $\mathrm{CFTR}^{31,32}$, contains a PDZBD at its carboxyl terminus (Appendix Fig. S1; see also Fig. 1a). Whole-mount in situ hybridization (WISH) detected both maternal and zygotic cftr expression throughout early development, with a relative enrichment in the branchial and pharyngeal arches at $24 \mathrm{hpf}$ (prim5 stage) (Appendix Fig. S2). This expression patterns suggest that Cftr plays a role in early axis formation in addition to previously reported Kupffer's vesicle (KV) development ${ }^{32}$.

We next generated a cftr frameshift mutant using TALENs technology ${ }^{32}$ (Appendix Fig. S3) and also observed the absent KV lumen phenotype at 8-somite stage in the mutant embryos as Navis et al. described ${ }^{32}$. Since a large percentage of homozygous $c f t r$ mutant larvae was lost beginning around $10 \mathrm{dpf}^{33}$, we examined heterozygous cftr mutant instead throughout the study. At 8somite stage $(13 \mathrm{hpf})$, the early hematopoietic precursor markers scl, gata2a, and $l m o 2$ and the primitive erythroid marker gata1a were markedly reduced in cftr mutant embryos (Fig. 2a). In contrast, the early vascular development markers flila (Fig. 2a), lateral mesoderm marker, draculin (Appendix Fig. S4), and the later vascular endothelium marker, $f l k 1$ (Appendix Fig. S5), were not changed significantly in $c f t r$ mutant. These results suggest a role of Cftr in zebrafish primitive hematopoiesis.

We next examined definitive hematopoiesis markers runx1 (30 hpf) and c-myb (48 hpf) and found their expression in $c f t r$ mutants was markedly decreased (Fig. 2b). Furthermore, the expression of myeloid progenitor marker pu.1 (30 hpf) and mature erythrocyte 


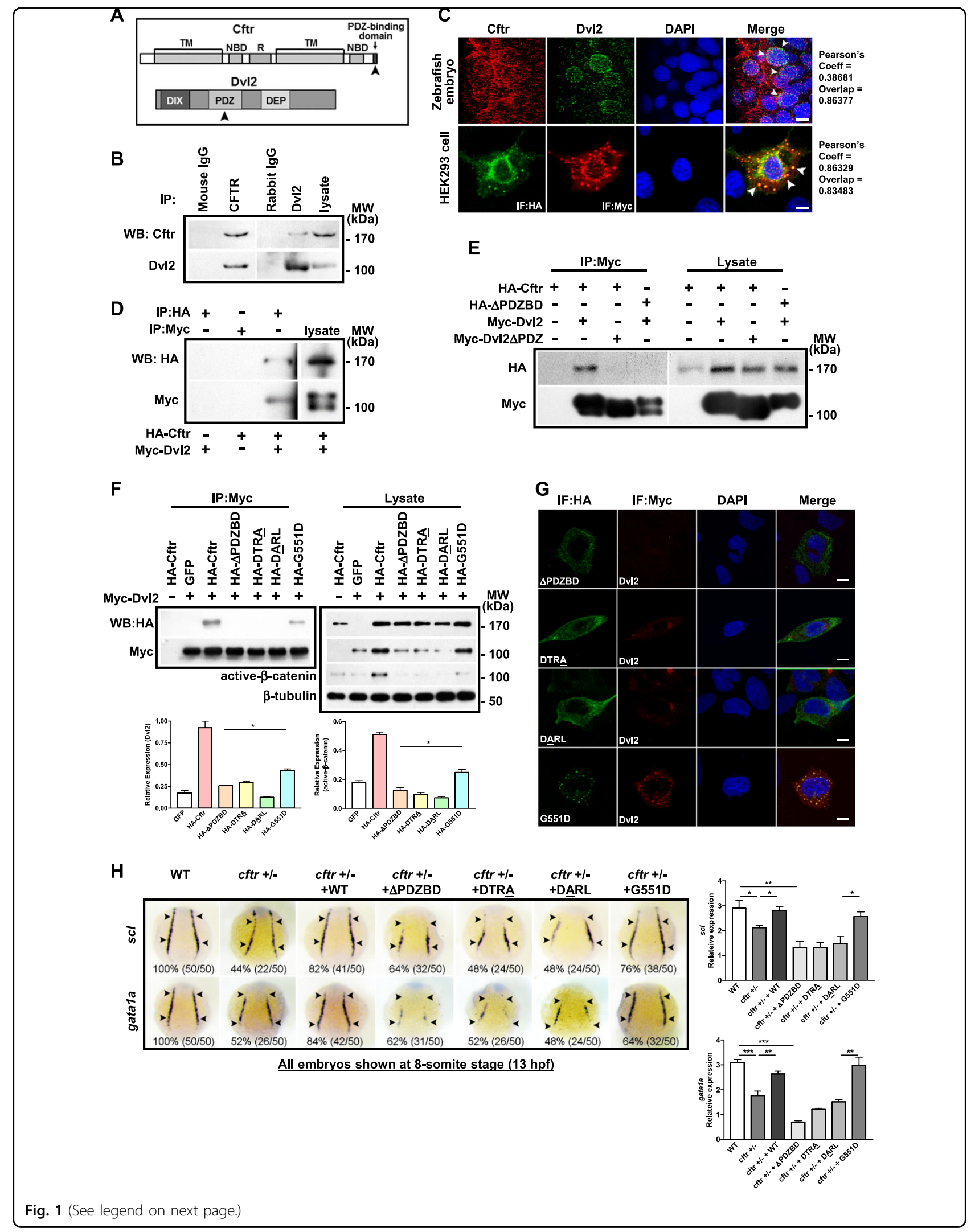




\begin{abstract}
(see figure on previous page)
Fig. 1 Cftr PDZBD but not its channel function is required for its interaction with Dvl2 and hematopoiesis. a Schematic drawing of PDZBD in Cftr and PDZ domain in Dvl2. Referring to Gee, H. Y. (2011)[57] and Wallingford, J. B. (2005)[58]. NBD nucleotide-binding domain, TM transmembrane domain, $\mathrm{R}$ regulatory domain, DIX Dishevelled and AXIN domain, DEP Dishevelled EGL-10 Pleckstrin domain. b Co-immunoprecipitation (Co-IP) of endogenous Dvl2 and Cftr in zebrafish embryos. c Co-localization of Cftr and Dvl2 in zebrafish embryo and HEK293 cells. Arrowheads indicate colocalized sites. Scale bar $5 \mu \mathrm{m}$. $\mathbf{d}$ In vitro binding assay identifies the physical interaction of Cftr with Dvl2. e Co-IP of exogenous Cftr and Dvl2 in HEK293 cells showing lack of interaction when Cftr PDZBD or DVI2 PDZ domain is deleted. f Co-IP showing the interaction of DVI2 with G551D, but not Cftr PDZBD mutants (either deletion or point mutation), and western blotting showing that Cftr and G551D overexpression increased the expression of DvI2 and active- $\beta$-catenin more significantly than that induced by Cftr PDZBD mutants. $\mathbf{g}$ Immunofluorescence analysis in HEK293 cells shows that either of the CFTR PDZBD mutants is not co-localized with Dvl2, whereas overexpressed G551D is co-localized with Dvl2. $\mathbf{h}$ Effects of different cftr mutants mRNA in rescuing hematopoietic defects in cftr mutant zebrafish embryos. Injection of cftr PDZBD mutants individually, with deletion or point mutation, could not rescue the hematopoietic defect in cftr mutant embryos. Injection of G551D rescues the hematopoietic defect in cftr mutant embryos. Embryos shown are dorsal views with anterior to the top at 8-somite stage (13 hpf)
\end{abstract}

marker hbbe3 (24 hpf) decreased dramatically in cftr mutant, suggesting that the development of erythroid and myeloid lineages was both impaired. Finally, the staining of O-dianisidine, a marker for red blood cells, was also reduced in cftr mutant embryos at $36 \mathrm{hpf}$ (Fig. 2b, bottom row), further indicating a severe defect in red blood cell formation. In addition, we also observed deficient expression of progenitor cell marker, $s c l$, erythroid marker, gata1a, and myeloid marker, l-plastin, in early larval stages (3 dpf) (Appendix Fig. S6). Similar hematopoietic defects were obtained using cftr antisense morpholino oligonucleotides (Appendix Figs. S6-10), which could be rescued by co-injection of human CFTR mRNA, demonstrating the specificity of the morpholinos and functional conservation of the CFTR between fish and human (Appendix Fig. S7). Furthermore, overexpressing zebrafish cftr mRNA led to the expansion of hematopoietic markers at 8-somite stage (Appendix Fig. S11). Collectively, these results provide evidence supporting that Cftr plays a critical and evolutionarily conserved role in primitive and definitive hematopoiesis.

\section{Cftr deficiency inhibits Wnt-dependent hematopoiesis}

Zebrafish hematopoietic progenitors emerge from the mesoderm at the gastrula stage ${ }^{29}$, and Wnt signaling plays a key role in this process ${ }^{20}$. We tested whether $\mathrm{Cftr}$ expression might affect Wnt-dependent hematopoiesis. Indeed, whereas injection of $1 \mathrm{pg}$ of wnt $3 a$ mRNA led to an expansion of scl and gatala in 54\% and 58\% of embryos, respectively, at the 8-somite stage (Fig. 3a), coinjection with equal amount of cftr tMO reduced the percentages of embryos with expansion of $s c l$ and gatala to $24 \%$ and $20 \%$, respectively (with $76 \%$ and $80 \%$ of embryos displaying no expansion of $\mathrm{scl}$ and gata1a, respectively, Fig. 3a). WISH results further revealed that the depletion of $c f t r$ either by TALENs or morpholinos also reduced the expression of $c d x 4$, hoxa $9 a, c-m y c$, and lef1 (Fig. 3b, c and Appendix Fig. S12), which are key Wnt targets known to play essential role in hematopoiesis ${ }^{20,34}$.
We further examined the effect of CFTR knockdown, induced by siRNA, on the Wnt3a-induced TopFlash luciferase activity in HEK293 cells and observed a significant reduction (Fig. 3d), confirming a role of CFTR in regulating Wnt signaling. Taken together, these results suggest an important role of CFTR in Wnt-dependent hematopoiesis.

\section{Dvl is required for CFTR-regulated Wnt-dependent hematopoiesis}

To further explore the role of Cftr in Wnt signaling during hematopoiesis, we further examined the effect of Cftr expression on key adaptors of the Wnt signaling, Dvls, in zebrafish. We found that the protein levels, but not mRNA level, of Dvl2 and Dvl3, were significantly reduced in cftr mutant and morphants at the beginning of gastrula period (5hpf) (Fig. 3e, f and Appendix Fig. S13). Furthermore, nuclear $\beta$-catenin was also significantly reduced in cftr mutant embryos or morphants (Fig. 3e and Appendix Fig. S13), consistent with a disruption of Wnt signaling. Similar to the findings in zebrafish, cotransfection of $H A-C f t r$ significantly increased protein levels of Dvl1-3, as well as nuclear $\beta$-catenin, in HEK293 cells transfected with $m y c-d v l 1-3$ (Fig. 3g), suggesting that $\mathrm{Cftr}$ contributes to the maintenance of Dvl protein levels, thereby sustaining Wnt signaling activation. Thus, it appears plausible that the impaired hematopoiesis in cftr mutant or morphants could be due to a deficiency in Dvl. Indeed, injection with $30 \mathrm{pg}$ of $d v l 2$ mRNA ameliorated the hematopoietic defect caused by deficient Cftr: percentages of embryos with normal $s c l$ and gatala expression pattern increased from $50 \%$ to $68 \%$, and from $52 \%$ and $74 \%$, respectively, in cftr mutant (Fig. 3h), and from $44 \%$ to $74 \%$, and from $24 \%$ to $88 \%$, respectively, in $c f t r$ morphants (Appendix Fig. S14). Concomitantly, active- $\beta$ catenin and Wnt target genes, $c$-myc and lef1, also showed recovered expression pattern in $c f t r$ mutant embryos injected with $d v l 2$ mRNA (Appendix Fig. S15). Similar rescuing effects of $d v l 2$ mRNA on definitive 


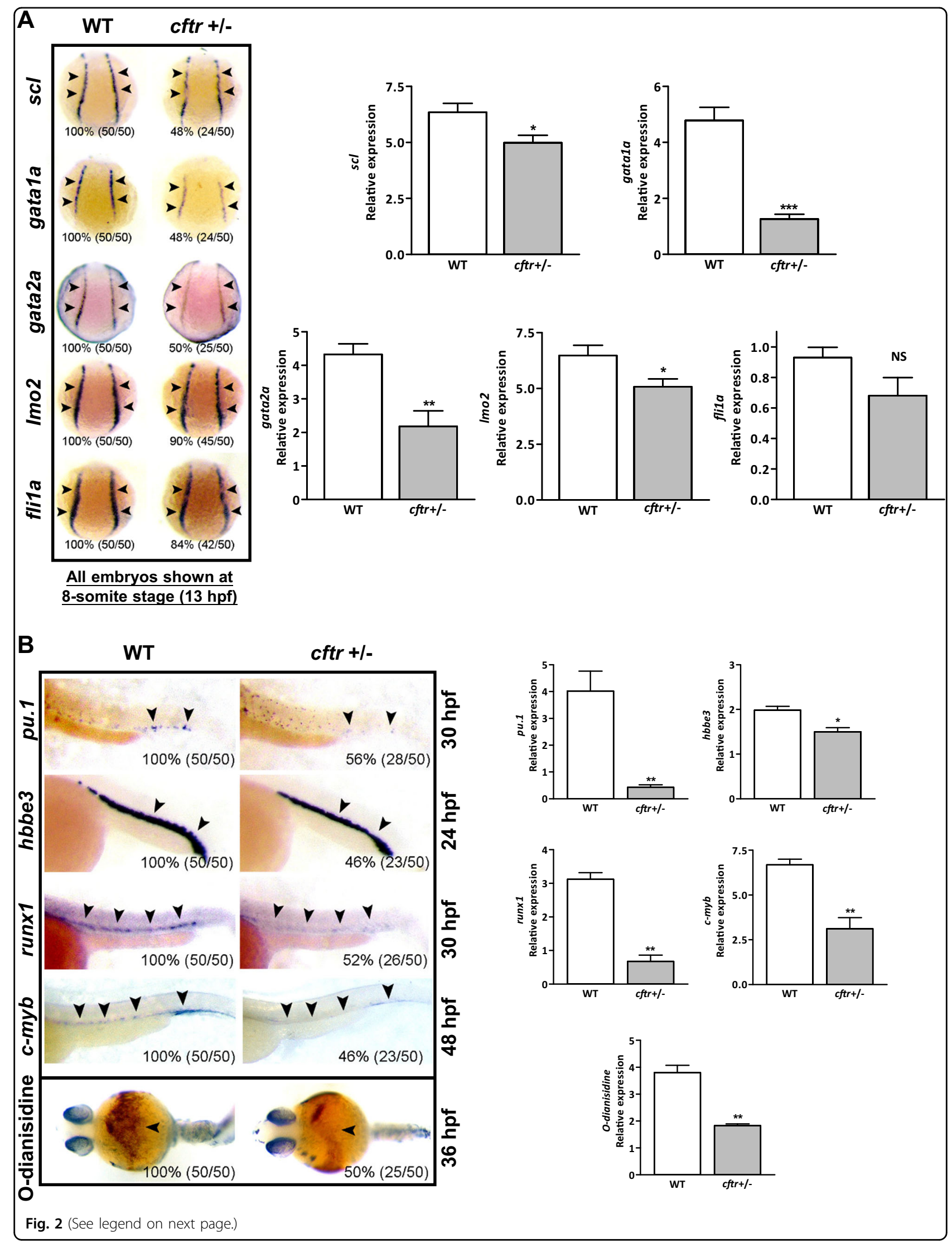


(see figure on previous page)

Fig. 2 Cftr critically regulates hematopoiesis in zebrafish. a cftr mutants show hematopoietic defects at 8-somite stage (13 hpf). Embryos shown are dorsal views with anterior oriented at the top. $\mathbf{b}$ cftr mutants show hematopoietic defects at later stages. Embryos shown are lateral views with anterior to the left. Bottom panel: cftr mutants displayed decreased hemoglobin staining by O-dianisidine. Embryos shown are ventral views with anterior to the left. Arrowheads indicate the expression sites of each marker gene. All genes were assayed by WISH. Histogram representing the relative expression detected by signal strength grayscale using software ImageJ at corresponding assay. The percentage and numbers indicated in each picture are the ratio for the number (left in bracket) of affected embryos with phenotype similar to what is shown in the picture and the total number (right in bracket) of observed embryos. The same number labeling was used thereafter

hematopoiesis markers, runx1 and $c-m y b$, were also observed at $28 \mathrm{hpf}$ and $48 \mathrm{hpf}$ (Appendix Fig. S16). These data suggest that defective Cftr results in a Dvl deficiency, and thus, an impaired Wnt-dependent hematopoiesis.

\section{Cftr functionally interacts with Dvl via PDZBD}

The reduced Dvl in $c f t r$ mutant or morphants suggested the importance of Cftr in stabilizing Dvl possibly through a direct protein-protein interaction. Reciprocal immunoprecipitation (co-IP) of endogenous Cftr and Dvl2 in zebrafish embryos at $5 \mathrm{hpf}$ (Fig. 1a, b) and mouse bone marrow (Appendix Fig. S17), which consists of primarily hematopoietic cells, was conducted to confirm this hypothesis. Furthermore, whole-mount immunostaining assay in zebrafish embryos revealed co-localization of CFTR with Dvl2, with the majority of the signals detected in the cytoplasm (Fig. 1c). Similarly, exogenously expressed Cftr and Dvl2 were co-localized in HEK293 cells (Fig. 1c). In addition, the interaction between $\mathrm{Cftr}$ and Dvl 2 was validated by in vitro protein binding assay (Fig. 1d).

To confirm the importance of PDZBD and PDZ domain in the interaction between Cftr and Dvl2, we constructed deletion mutants of Cftr and Dvl2 lacking PDZBD and PDZ domain, respectively. Co-IP in HEK293 cells showed that either mutation abrogated the interaction between these two proteins (Fig. 1e), suggesting the requirement of both PDZBD and PDZ for Cftr-Dvl2 interaction. To provide further evidence for the involvement of the PDZBD of Cftr in its interaction with Dvl2, we generated two additional mutants, DTRA and DARL, each carrying a point mutation in PDZBD as described by Moyer et al. $^{35}$. Similar to Cftr PDZBD deletion mutant, both point mutations failed to bind to Dvl2 and enhance the expression of Dvl2 and active $\beta$-catenin in HEK293 cells (Fig. 1f, g). We next compared the ability of $c f t r$ WT and cftr PDZBD mutants to rescue the hematopoietic defect in cftr mutant embryos. Whereas injection with $50 \mathrm{pg}$ of wild-type (WT) cftr mRNA increased percentages of $c f t r$ embryos with normal $s c l$ and gatala expression level from $56 \%$ to $82 \%$, and from $48 \%$ to $84 \%$, respectively (Fig. $1 \mathrm{~h}$ ), injection with $50 \mathrm{pg}$ of mRNA of any $c$ ftr PDZBD mutant individually failed to rescue the hematopoietic defect in cftr mutant-the percentages of embryos with abnormal scl and gatala expression level were not significantly changed after the injection (Fig. 1h). Similar results were also observed for definitive hematopoiesis markers, runx 1 and $c-m y b$, at $28 \mathrm{hpf}$ and $48 \mathrm{hpf}$, confirming the importance of PDZBD in the Cftr-regulated hematopoiesis (Appendix Fig. S18).

\section{PDZBD but not CFTR channel function is required for its interaction with Dvl}

G551D is a well-known mutation causing gating defect in CFTR channel function ${ }^{36,37}$. To address whether the regulation of Wnt signaling in hematopoiesis by CFTR is independent of its channel gating function, we tested G551D mutant which has an intact PDZBD. CFTR G551D was able to bind to Dvl2 and significantly enhance the expression of Dvl2 and active- $\beta$-catenin in HEK293 cells (Fig. 1f, g). Consistently, 50 pg of G551D mRNA recovered the expression of scl/gatala (from $56 \%$ to $76 \%$, and from 485 to $64 \%$, respectively) and runx $1 / c-m y b$ (from $42 \%$ to $64 \%$, and from $44 \%$ to $68 \%$, respectively) in cftr mutant embryos (Fig. 1h and Appendix Fig. S18). Collectively, the PDZBD of Cftr, but not its channel function, is essential for maintaining a stable level of Dvl2 protein and Wnt signaling for hematopoiesis.

\section{Cftr deficiency results in accelerated Dpr1-induced lysosomal degradation of Dvl2}

The stability of Dvl is regulated by either proteasomal, or lysosomal, or autophagic degradation pathway ${ }^{21,38}$. Our CHX-chase assays using zebrafish embryos demonstrated Dvl2 degradation in cftr mutant (Fig. 4a) and morphant (Appendix Fig. S19A) embryos. Then, we used specific inhibitors for these degradation pathways to identify the mechanism for Dvl degradation in cftr mutant and morphants. We found that the reduction of Dvl2 in cftr mutant (Fig. 4b) and morphants (Appendix Fig. S19B) could be rescued by lysosome inhibitor $\mathrm{NH} 4 \mathrm{Cl}(\mathrm{NC})$, but not proteasome inhibitor MG132 or autophagy inhibitor 3-methyladenine (3-MA). These results suggest that deficiency of $\mathrm{Cftr}$ results in lysosomal degradation of Dvl2.

We next explored the mechanism by which Cftr prevents lysosomal degradation of Dvl. Since Dpr1 by interacting with Dvl promotes Dvl degradation in lysosomes ${ }^{24}$, 


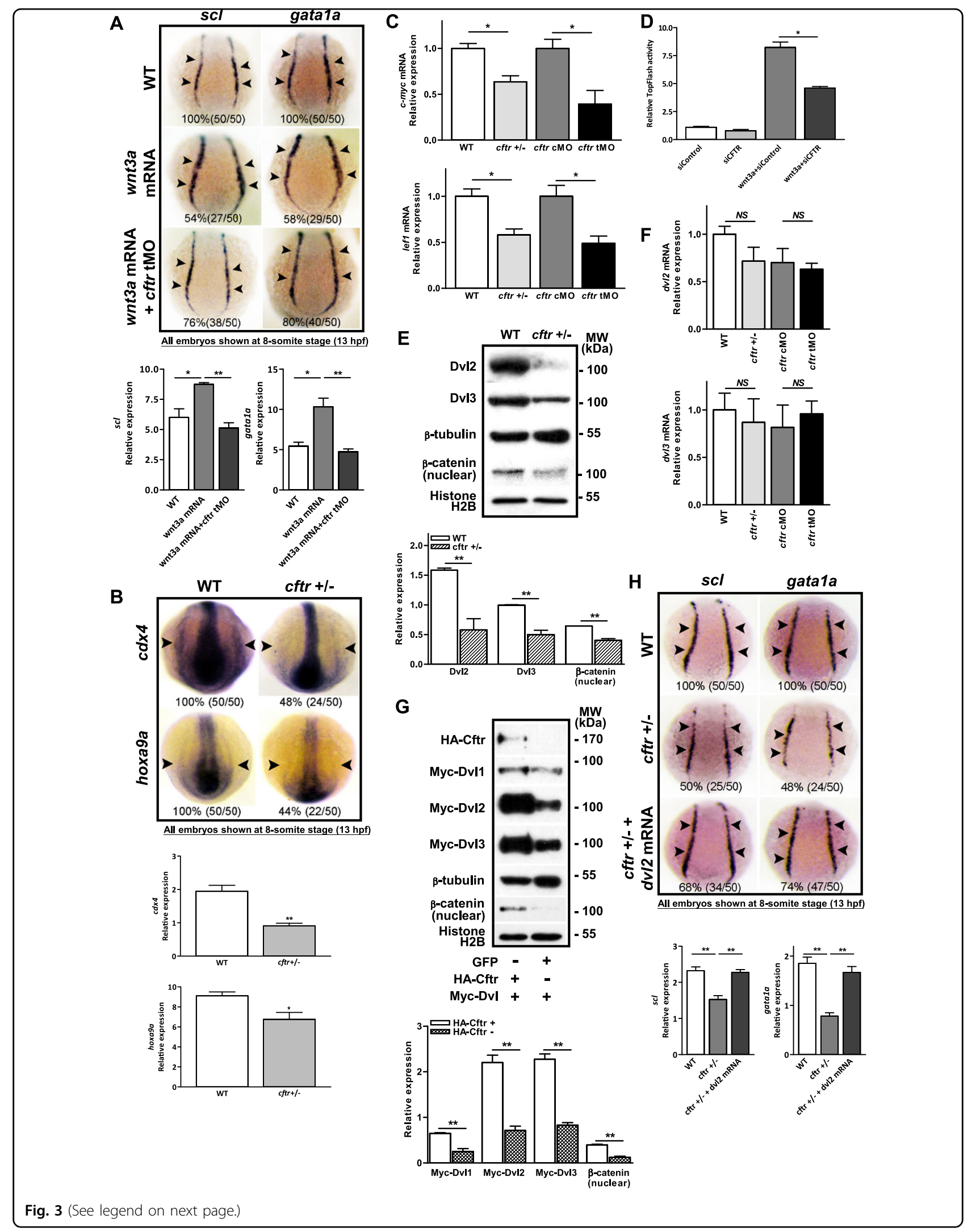


(see figure on previous page)

Fig. 3 Involvement of Cftr in Wnt-dependent hematopoiesis in zebrafish. WISH showing impaired wnt3a-induced expression of scl and gatala in wht3a mRNA and cftr morpholino co-injected zebrafish (a) and decreased expression of cdx4 and hoxa9a in cftr mutants (b). c Quantitative RT-PCR showing significantly reduced c-myc and lef1 mRNA levels in cftr mutants. $\mathbf{d}$ CFTR knockdown by siRNA attenuated the Wnt reporter TopFlash activity in HEK293 cells $(n=3)$. e Western blot analysis showing reduced Dvl2, 3 and nuclear $\beta$-catenin expression in cftr mutants. f Quantitative RT-PCR showing no significant changes in dvl2, 3 mRNA levels in cftr mutants. $\mathbf{g}$ Western blot analysis showing increased Dvl1-3 protein levels in coexpressing Cftr with Dvl1-3 HEK293 cells as compared to Dvl1-3 alone controls. $\mathbf{h}$ Effects of dv/2 mRNA in rescuing hematopoietic defects in cftr mutants. Embryos shown in $\mathbf{a}, \mathbf{b}$, and $\mathbf{h}$ are dorsal views with anterior to the top at 8-somite stage (13 hpf)
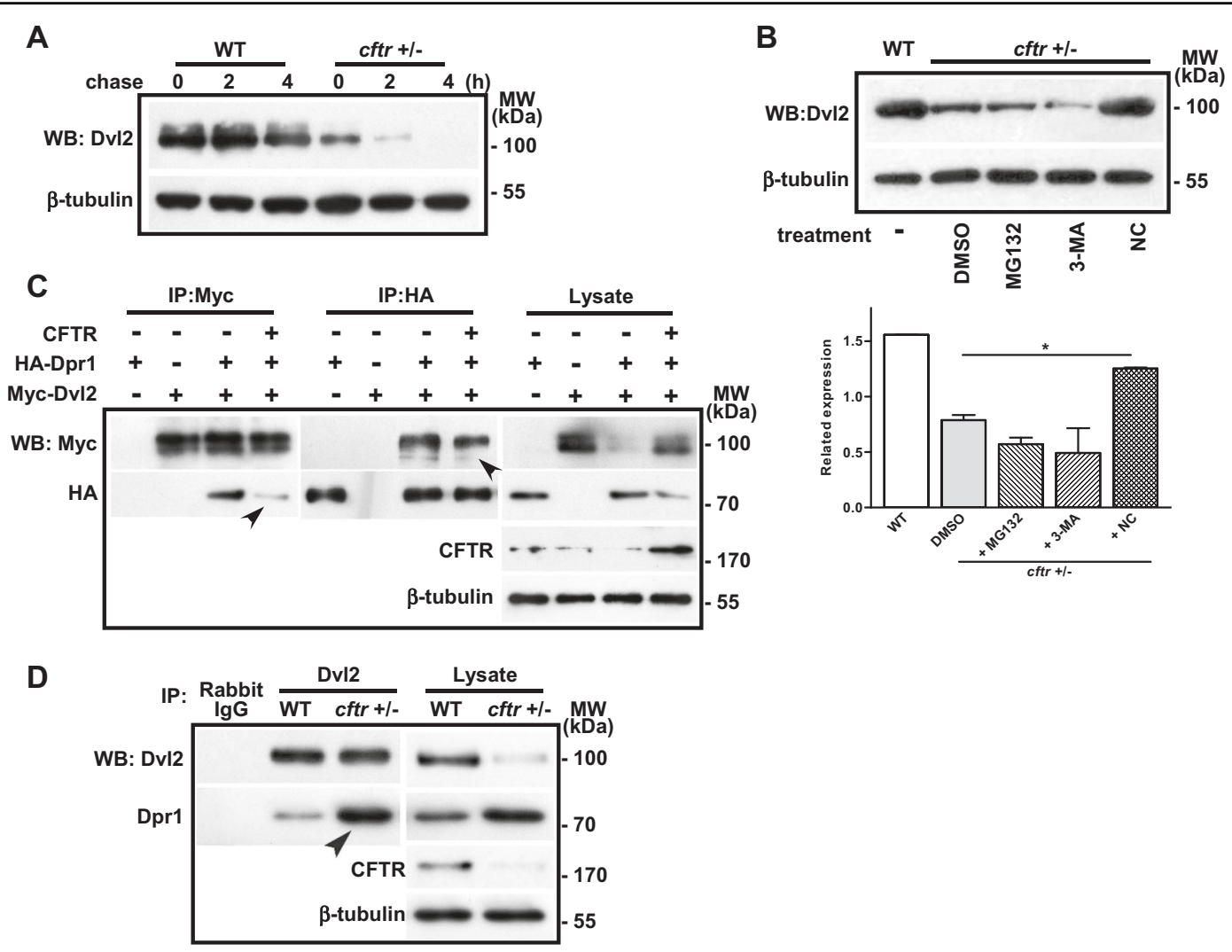

Fig. 4 Cftr prevents Dvl2 degradation through DPR1-induced lysosomal pathway. a Zebrafish embryos at 5 hpf were CHX chased at the indicated time (in hours). Dvl2 shows degradation pattern in cftr mutant zebrafish embryos. The protein levels were analyzed by western blot. $\mathbf{b}$ Lysosomal inhibitor (NC), but not proteasome inhibitor (MG132) or autophagy inhibitor (3-MA) recovers Dvl2 expression in cftr mutant zebrafish embryos. c Co-IP/western blot analysis in HEK293 cells shows that Dvl2 interacts with DPR1, and CFTR overexpression decreases the protein-protein interaction between Dvl2 and DPR1 (marked by arrowheads). d Co-IP/western blot analysis in zebrafish embryos shows that endogenous Dvl2 interacts with DPR1, and cftr mutants increases the protein-protein interaction between Dvl2 and DPR1 (marked by arrowheads)

we asked whether Cftr may impart its protective effect by attenuating Dpr1-Dvl interaction. Indeed, Co-IP results revealed that exogenous $\mathrm{Cftr}$ abrogated the interaction of Dvl2 with DPR1 in HEK293 cells (Fig. 4c). Consistently, we detected increased Dpr1 protein level bound by Dvl2 in cftr mutant embryos (Fig. 4d). Taken together, these data suggest that Cftr interferes with the interaction between Dpr1 and Dvl, thus preventing Dpr1-induced Dvl degradation through lysosomal pathway.
CFTR correlates with DVL, $\beta$-CATENIN, and hematopoietic factors in human blood system

Since Wnt signaling and hematopoietic factors are evolutionally conserved in vertebrates, the currently demonstrated CFTR involvement in Wnt-dependent hematopoiesis in zebrafish prompted us to examine whether the expression levels of CFTR are correlated with Wnt signaling and related hematopoietic factors in humans. By searching the database R2: Genomics 


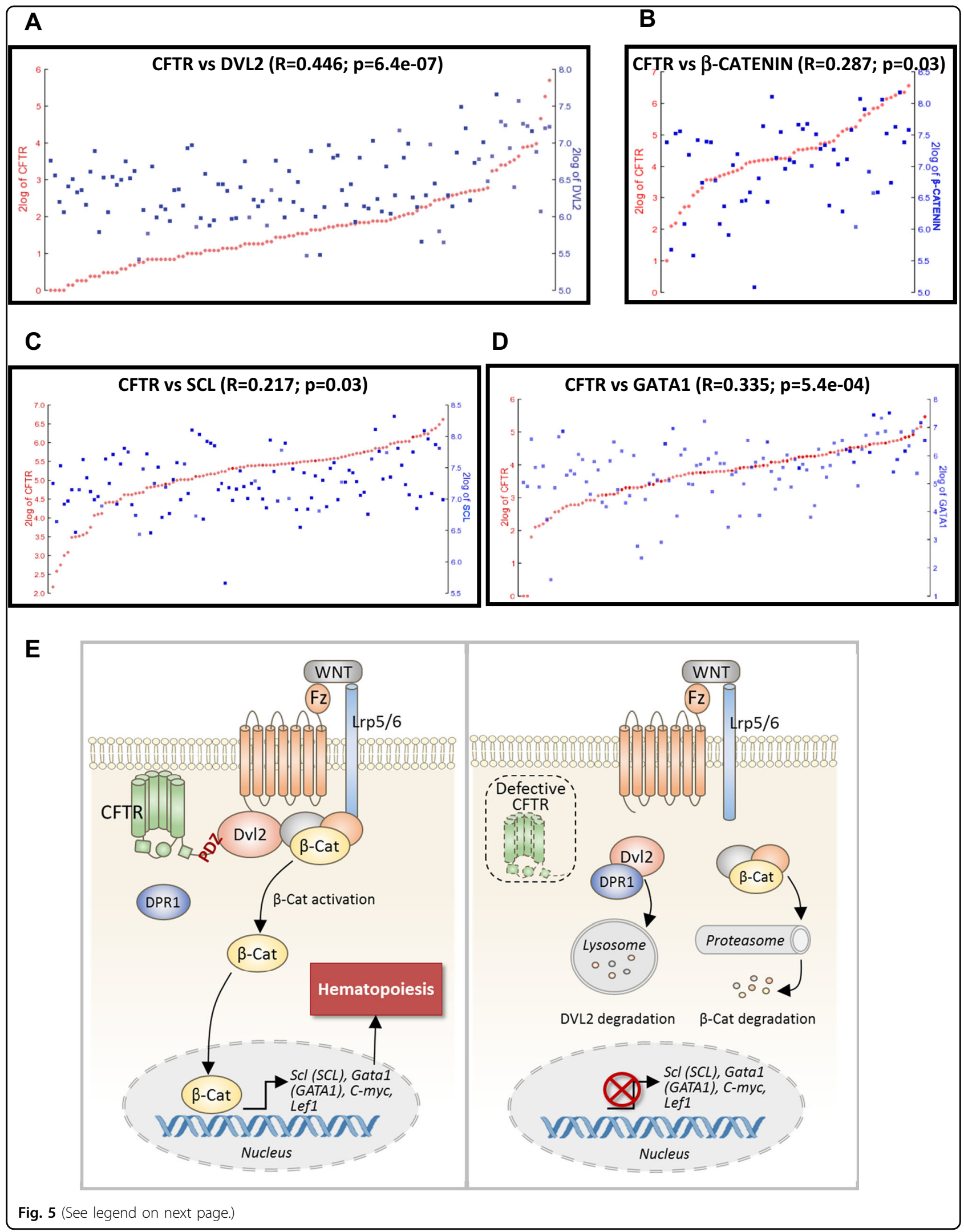


(see figure on previous page)

Fig. 5 CFTR correlates with DVL2 and key hematopoietic factors in human blood system. R2: Genomics Analysis and Visualization Platform (http://r2.amc.nl) shows that CFTR expression levels positively correlate with DVL2 in human normal leukocytes (a), $\beta$-CATENIN in human bone marrows (b), SCL (c), and GATA1 (d) in mixed human blood. e Model for regulation of Wnt signaling by Cftr in hematopoiesis. Left panel: Cftr (CFTR) binds to DVl2 (DVL2) through PDZBD and prevents DVI2(DVL2) from Dpr1-induced lysosomal degradation leading to the activation of Wnt signaling and transcription of hematopoietic target genes in zebrafish and human (in capital). Right panel: defective Cftr (CFTR) results in accelerated Dpr1induced DVI2(DVL2) degradation, and thus $\beta$-catenin ( $\beta$-CATENIN) complex degradation, leading to inactivation of Wnt signaling and impaired hematopoiesis. Fz frizzled class receptors, $\beta$-cat $\beta$-catenin

Analysis and Visualization Platform (http://r2.amc.nl), we found that CFTR is positively correlated with DVL2 $(n=$ 114 , Fig. $5 \mathrm{a}$ ), $\beta$-CATENIN ( $n=56$ Fig. $5 \mathrm{~b}$ ), as well as hematopoietic factors, SCL $(n=105$, Fig. $5 \mathrm{c})$ and GATA1 $(n=103$, Fig. $5 d)$ in human blood system, confirming an important role of CFTR in Wnt-dependent hematopoiesis in humans.

\section{Discussion}

While CFTR has been implicated in the organogenesis of the lung ${ }^{39}$ and gut/intestine ${ }^{40}$ with its potential role suggested in regulating Wnt signaling in these developmental contexts, detailed mechanisms have not been elucidated. A recent study using novel deep proteomic analysis has identified a close association of CFTR with the components of Wnt/ $\beta$-catenin pathway among 638 individual high-confidence CFTR interactors ${ }^{41}$. The present study has provided the first evidence for the involvement of CFTR in the regulation of Wnt-dependent hematopoiesis through its interaction with and regulation of the degradation process of a key adaptor protein of Wnt signaling, Dvl (Fig. 5e).

During primitive hematopoiesis, Wnt cooperates with BMP signaling to promote blood fate through activation of the Cdx-Hox-Scl/Gatala genetic pathway in embryonic stem cells and zebrafish embryo ${ }^{19,20}$. During definitive hematopoiesis, Wnt signaling is involved in Prostaglandin E2-regulated pathway to drive specification of $\mathrm{HSCs}^{42}$. Although most studies have found a positive role for Wnt pathway in HSCs during development ${ }^{26}$, the importance of Wnt signals in normal hematopoiesis is less conclusive $^{43,44}$. The present study demonstrates a crucial role of Wnt signaling in primitive and definitive hematopoiesis, and in addition, we illustrate that the activity of Wnt pathway in this developmental context is regulated by CFTR, a heretofore unknown regulator of hematopoiesis. Furthermore, our findings may provide a tangible etiology for the severe anemia seen in some CF patients ${ }^{17}$, which may be due to a compromised hematopoiesis caused by defective CFTR-dependent Wnt signaling.

Of note, a previous study by Navis et al. ${ }^{32}$ has detected not only the absence of KV but also a defect in the ventral lateral region of the embryo, where hematopoietic progenitor cells emerge, in 10-somite stage $c f t r$ mutant. This is consistent with the hematopoietic defect evidenced by the reduction of hematopoietic markers observed in this study.

However, despite a remarkable effect on hematopoiesis observed in cftr mutants, from both the current and previous studies ${ }^{32}$, the effects of $c f t r$ mutation/knockdown on the Wnt signaling target genes are relatively mild (Fig. 3b-d). Interestingly, Luis et al. ${ }^{45}$ also reported that canonical Wnt signaling regulates hematopoiesis in a dosage-dependent fashion. They found that only a mild level of activation of Wnt signaling was able to enhance HSC function, whereas, intermediate and high level of activation of Wnt signaling impaired HSC self-renewal or differentiation. However, using Wnt3a-I- mice, the same group demonstrated that Wnt3a deficiency leads to a depletion of the HSC pool, similar to that found with intermediate and high level of Wnt activation ${ }^{46}$. Our finding of a relatively mild effect on Wnt signaling target genes in cftr mutation/knockdown zebrafish (Fig. 3c-e) is consistent with the previous findings ${ }^{45,46}$ and supports the notion that hematopoiesis is regulated by a delicate balance of Wnt signaling levels. Therefore, the variation in Wnt signaling levels in different studies might account for the apparent contradictory findings in mouse $\mathrm{HSCs}^{43,44}$.

Although CFTR has been shown to interact and regulate a number of PDZ-containing proteins, including several ion channels ${ }^{13}$ and transporters ${ }^{47,48}$, as well as adaptor proteins that interact with other signaling proteins $^{49}$, it remains an open question whether CFTR channel activity is required for its regulatory role. Most studies used CFTR channel blockers, such as CFTRinh172 , and observed a reversed, reduced, or impaired regulatory effect of CFTR, and interpreted these results as an indication that CFTR channel activity is required for the regulatory action of CFTR. However, these channel blockers have non-specific effects ${ }^{50}$, making the results inconclusive. In the present study, we show that mutations in PDZBD disrupt Cftr-Dvl interaction and fail to support Wnt signaling in zebrafish; however, PDZBDintact G551D mutation in NBD1, which has been shown to produce CFTR chloride channel defect ${ }^{36,37}$, retains the ability of Cftr to interact with Dvl and rescues Wnt signaling in cftr mutant embryos. These results provide compelling evidence for a regulatory role of CFTR that is 
dependent on its PDZ-binding activity but independent of its channel function. This is consistent with the finding from a previous study of the regulatory effect of CFTR on chemokine RANTES expression in the airways ${ }^{51}$. The authors of this earlier study also noted that the ability of CFTR to restore RANTES expression is not entirely dependent on the amount of CFTR inserted into the plasma membrane. In the present study, we also show that most of the CFTR-Dvl interaction takes place in the cytoplasm, but not at the plasma membrane (Fig. 3c, g), further indicating that this interaction is independent of CFTR channel activity, a function that requires the insertion of CFTR into the plasma membrane.

Our study also provides the first evidence that CFTR can interfere with DPR1-Dvl interaction thereby preventing DPR1-induced lysosomal Dvl degradation. We propose that CFTR competes with DPR1 for binding to Dvl and stabilizes Dvl to maintain a normal level of Wnt signaling required for hematopoiesis (Fig. 4c, d).

The genomic analysis of human blood system database in the present study has also lent strong support for an important role of CFTR in regulating Wnt-dependent hematopoiesis in humans, as demonstrated in the zebrafish model. Not only is CFTR positively correlated with DVL2, but also $\beta$-CATENIN as well as the key hematopoietic factors, SCL and GATA, downstream of the Wnt signaling in human blood system (Fig. 5a-d), consistent with an evolutionally conserved role in CFTR in Wntregulated hematopoiesis. Given that Wnt signaling plays an important role in hematopoiesis, the presently demonstrated conserved role of CFTR in regulating Wnt signaling suggests its possible involvement in the pathogenesis of different forms of hematopoietic disorders/ diseases in humans apart from anemia in CF. Therefore, CFTR may be a potential target for diagnosis and treatment of related hematopoietic diseases.

\section{Materials and methods}

\section{TALEN-mediated CFTR mutagenesis in zebrafish}

The TALENs plasmid pair was kindly provided by Dr. Michel Bagnat (Duke University), and the mutagenesis was performed as Navis et al. described ${ }^{32}$. Differently, we used T7 Endonuclease I assays ${ }^{52}$ (New England Biolabs) instead of EcoRV digestion to identify the mutants. The cftr heterozygous mutant embryos were obtained by mating $c f t r$ heterozygous mutant male fishes with wildtype female fishes.

\section{Zebrafish embryo manipulation, histology, and in situ hybridization}

WISH was carried out as previously described in Thisse et al. $^{53}$ and Sun et al. ${ }^{54}$. After lineage by appropriate restriction enzymes, antisense RNAs for in situ hybridization were synthesized using DIG RNA Labeling Kit
(SP6/T7) (Roche) and purified by MEGAclear (Ambion). Two non-overlapping probes, targeting to the sequence 35-1027 bp and 3400-4562 bp, were designed for cftr in situ hybridization detection.

Immunofluorescence in zebrafish embryos was performed as previously described (Jia et al. ${ }^{55}$ and Brend et $\mathrm{al}^{56}$ ) with modifications. Embryos were fixed in fresh $2 \%$ paraformaldehyde overnight, permeabilized in $100 \%$ methanol at $-20^{\circ} \mathrm{C}$ for at least $1 \mathrm{~h}$. The embryos were bathed in $1 \mathrm{mM}$ EDTA (pH 8.0) at $94-100^{\circ} \mathrm{C}$ for $10 \mathrm{~min}$ and cooled down to room temperature, then incubated in block solution (PBS plus $0.5 \%$ Triton X-100 and 1\% BSA) for $1 \mathrm{~h}$ at room temperature. Embryos were then incubated with a primary antibody at $4{ }^{\circ} \mathrm{C}$ overnight, followed by incubation with secondary antibodies (Alexa Fluor 488 and 594 IgG from Life Technologies Corporation) at room temperature for $1 \mathrm{~h}$. The nuclei were stained by DAPI. The embryos were photographed with Olympus_FV1000 laser scanning confocal microscope.

For immunofluorescence in cells, adherent cells grown on coverslips. All cells were fixed with fresh $4 \%$ formaldehyde for $10 \mathrm{~min}$ at room temperature, then permeabilized by $0.3 \%$ Triton X-100 in PBS for $15 \mathrm{~min}$ and blocked with $1 \%$ BSA and $0.3 \%$ Triton X-100 in PBS buffer. The cells were then probed with primary antibodies, followed by Alexa Fluor 488/546/594 secondary antibodies (Life Technologies Corporation). Fluorescence images were acquired with Olympus_FV1000 laser scanning confocal microscope.

O-dianisidine staining was performed as described by Yue et $\mathrm{al}^{27}$.

Other Materials and Methods are given as Supplementary Information.

\footnotetext{
Acknowledgements

The authors wish to thank Dr. Michel Bagnat (Duke University) for kindly providing cftr TALEN plasmid pair; Prof. Chen Ye-Guang (Tsinghua University) for kindly providing HA-Dpr1 plasmid; Dr. Kin Lam Fok for his work in cftr sMO analysis; Dr. Ye Chun Ruan for her art work in the manuscript; Dr. Junjiang Chen for his help in big data analysis; Dr. Mei Zeng (North Sichuan Medical College and NIH), Dr. Sizhou Huang (ChengDu Medical College), Prof. Lingfei Luo (Southwest University), and Prof. Wing Simon (Mcgill University) for helpful discussion and suggestions. This research was supported by grants from National Natural Science Foundation of China (81200339), National Natural Science Foundation of China (81571390), National Basic Research Program of China (2013CB967404), China Postdoctoral Science Foundation (20110491723), Young Teacher foundation of Sichuan University (2011SCU11040), Focused Investment Scheme of The Chinese University of Hong Kong and K.S. Lo Foundation.
}

\section{Author details}

${ }^{1}$ SCU-CUHK Joint Laboratory for Reproductive Medicine, Key Laboratory of Birth Defects and Related Diseases of Women and Children (Sichuan

University), Ministry of Education, Department of Pediatrics, West China Second University Hospital, Sichuan University, 610041 Chengdu, China. ${ }^{2}$ Epithelial Cell Biology Research Center, School of Biomedical Sciences, Faculty of Medicine, The Chinese University of Hong Kong, Hong Kong SAR, China. ${ }^{3}$ Prenatal Diagnosis Center, Department of Obstetrics \& Gynecologic, West China Second University Hospital, Sichuan University, 610041 Chengdu, China. ${ }^{4}$ Department 
of Anatomical and Cellular Pathology, Faculty of Medicine, The Chinese University of Hong Kong, Hong Kong, China

\section{Author contributions}

H.S. and H.C.C. conceived and designed the experiments; H.S., Y.W., J.Z., Y.C., and K.S. performed the experiments, with the assistance of H.L.; H.S. and Y.L. analyzed the data; M.M., W.X., X.J., and X.Z. coordinated the project. H.S., H.C.C., Y.W., and W.X. wrote the paper.

\section{Conflict of interest}

The authors declare that they have no conflict of interest.

\section{Publisher's note}

Springer Nature remains neutral with regard to jurisdictional claims in published maps and institutional affiliations.

Supplementary Information accompanies this paper at (https://doi.org/ 10.1038/s41419-018-0311-9).

Received: 13 November 2017 Revised: 4 January 2018 Accepted: 8 January 2018

Published online: 15 February 2018

\section{References}

1. Gadsby, D. C., Vergani, P. \& Csanady, L. The ABC protein turned chloride channel whose failure causes cystic fibrosis. Nature 440, 477-483 (2006).

2. Tizzano, E. F., Chitayat, D. \& Buchwald, M. Cell-specific localization of CFTR mRNA shows developmentally regulated expression in human fetal tissues. Hum. Mol. Genet. 2, 219-224 (1993).

3. Riordan, J. R. CFTR function and prospects for therapy. Annu. Rev. Biochem. 77, 701-726 (2008)

4. Quinton, P. M. Physiological basis of cystic fibrosis: a historical perspective. Physiol. Rev. 79(1 Suppl), S3-S22 (1999).

5. Johannesson, B., Hirtz, S., Schatterny, J., Schultz, C. \& Mall, M. A. CFTR regulates early pathogenesis of chronic obstructive lung disease in betaENaCoverexpressing mice. PLOS ONE 7, e44059 (2012).

6. Pezzulo, A. A. et al. Reduced airway surface $\mathrm{pH}$ impairs bacterial killing in the porcine cystic fibrosis lung. Nature 487, 109-113 (2012).

7. Wilschanski, M. \& Novak, I. The cystic fibrosis of exocrine pancreas. Cold Spring Harb. Perspect. Med. 3, a009746 (2013).

8. Guo, J. H. et al. Glucose-induced electrical activities and insulin secretion in pancreatic islet beta-cells are modulated by CFTR. Nat. Commun. 5, 4420 (2014).

9. Xu, W. M. et al. Cystic fibrosis transmembrane conductance regulator is vital to sperm fertilizing capacity and male fertility. Proc. Natl Acad. Sci. USA 104, 9816-9821 (2007).

10. Lu, Y. C. et al. CFTR mediates bicarbonate-dependent activation of miR-125b in preimplantation embryo development. Cell Res. 22, 1453-1466 (2012).

11. Chen, $\mathrm{H}$. et al. Impaired CFTR-dependent amplification of FSH-stimulated estrogen production in cystic fibrosis and PCOS. J. Clin. Endocrinol. Metab. 97, 923-932 (2012).

12. Li, C. \& Naren, A. P. Analysis of CFTR interactome in the macromolecular complexes. Methods Mol. Biol. 741, 255-270 (2011)

13. Kunzelmann, K. CFTR: interacting with everything? News Physiol. Sci. 16 167-170 (2001).

14. Fan, J. S. \& Zhang, M. Signaling complex organization by PDZ domain proteins. Neurosignals 11, 315-321 (2002)

15. Sun, T. T. et al. Disrupted interaction between CFTR and AF-6/afadin aggravates malignant phenotypes of colon cancer. Biochim. Biophys. Acta 1843, 618-628 (2014).

16. Ruan, Y. C. et al. CFTR interacts with ZO-1 to regulate tight junction assembly and epithelial differentiation through the ZONAB pathway. J. Cell Sci. 127(Pt 20), 4396-4408 (2014)

17. Kahre, T., Teder, M., Panov, M. \& Metspalu, A. Severe CF manifestation with anaemia and failure to thrive in a 394delTT homozygous patient. J. Cyst. Fibros. 3, 58-60 (2004).
18. Luis, T. C., Ichii, M., Brugman, M. H., Kincade, P. \& Staal, F. J. Wnt signaling strength regulates normal hematopoiesis and its deregulation is involved in leukemia development. Leukemia 26, 414-421 (2012).

19. Ellett, F. \& Lieschke, G. J. Zebrafish as a model for vertebrate hematopoiesis. Curr. Opin. Pharmacol. 10, 563-570 (2010).

20. Lengerke, $\mathrm{C}$. et al. BMP and Wnt specify hematopoietic fate by activation of the Cdx-Hox pathway. Cell Stem Cell 2, 72-82 (2008).

21. Gao, C. \& Chen, Y. G. Dishevelled: the hub of Wnt signaling. Cell. Signal. 22 717-727 (2010).

22. Chang, B. et al. Epsin is required for Dishevelled stability and Wnt signalling activation in colon cancer development. Nat. Commun. 6, 6380 (2015).

23. de Groot, R. E. et al. Huwe1-mediated ubiquitylation of dishevelled defines a negative feedback loop in the Wnt signaling pathway. Sci. Signal 7, ra26 (2014).

24. Zhang, L., Gao, X., Wen, J., Ning, Y. \& Chen, Y. G. Dapper 1 antagonizes Wnt signaling by promoting dishevelled degradation. J. Biol. Chem. 281, 8607-8612 (2006)

25. Ma, B. et al. The Wnt signaling antagonist Dapper1 accelerates Dishevelled2 degradation via promoting its ubiquitination and aggregate-induced autophagy. J. Biol. Chem. 290, 12346-12354 (2015).

26. Jagannathan-Bogdan, M. \& Zon, L. I. Hematopoiesis. Development 140, 2463-2467 (2013)

27. Yue, R. et al. Beta-arrestin1 regulates zebrafish hematopoiesis through binding to YY1 and relieving polycomb group repression. Cell 139, 535-546 (2009).

28. Li, X., Jia, S., Wang, S., Wang, Y. \& Meng, A. Mta3-NuRD complex is a master regulator for initiation of primitive hematopoiesis in vertebrate embryos. Blood 114, 5464-5472 (2009)

29. Paik, E. J. \& Zon, L. I. Hematopoietic development in the zebrafish. Int. J. Dev. Biol. 54, 1127-1137 (2010).

30. de Jong, J. L. \& Zon, L. I. Use of the zebrafish system to study primitive and definitive hematopoiesis. Annu. Rev. Genet. 39, 481-501 (2005).

31. Bagnat, $M$. et al. Cse1l is a negative regulator of CFTR-dependent fluid secretion. Curr. Biol. 20, 1840-1845 (2010).

32. Navis, A., Marjoram, L. \& Bagnat, M. Cftr controls lumen expansion and function of Kupffer's vesicle in zebrafish. Development 140, 1703-1712 (2013).

33. Navis, A. \& Bagnat, M. Loss of cftr function leads to pancreatic destruction in larval zebrafish. Dev. Biol. 399, 237-248 (2015).

34. Shimizu, T., Bae, Y. K., Muraoka, O. \& Hibi, M. Interaction of Wht and caudalrelated genes in zebrafish posterior body formation. Dev. Biol. 279, 125-141 (2005).

35. Moyer, B. D. et al. The PDZ-interacting domain of cystic fibrosis transmembrane conductance regulator is required for functional expression in the apical plasma membrane. J. Biol. Chem. 275, 27069-27074 (2000).

36. Welsh, M. J. \& Smith, A. E. Molecular mechanisms of CFTR chloride channe dysfunction in cystic fibrosis. Cell 73, 1251-1254 (1993).

37. Hwang, T. C. \& Kirk, K. L. The CFTR ion channel: gating, regulation, and anion permeation. Cold Spring Harb. Perspect. Med. 3, a009498 (2013).

38. Gao, C. et al. Autophagy negatively regulates Wnt signalling by promoting Dishevelled degradation. Nat. Cell Biol. 12, 781-790 (2010).

39. Cohen, J. C., Larson, J. E., Killeen, E., Love, D. \& Takemaru, K. CFTR and Wnt/betacatenin signaling in lung development. BMC Dev. Biol. 8, 70 (2008).

40. Paul, T., Li, S., Khurana, S., Leleiko, N. S. \& Walsh, M. J. The epigenetic signature of CFTR expression is co-ordinated via chromatin acetylation through a complex intronic element. Biochem. J. 408, 317-326 (2007).

41. Pankow, S. et al. F508 CFTR interactome remodelling promotes rescue of cystic fibrosis. Nature 528, 510-516 (2015).

42. Goessling, W. et al. Genetic interaction of PGE2 and Wnt signaling regulates developmental specification of stem cells and regeneration. Cell 136, 1136-1147 (2009).

43. Reya, T. et al. A role for Wnt signalling in self-renewal of haematopoietic stem cells. Nature 423, 409-414 (2003).

44. Kirstetter, P., Anderson, K., Porse, B. T., Jacobsen, S. E. \& Nerlov, C. Activation of the canonical Wnt pathway leads to loss of hematopoietic stem cell repopulation and multilineage differentiation block. Nat. Immunol. 7, 1048-1056 (2006).

45. Luis, T. C. et al. Canonical wnt signaling regulates hematopoiesis in a dosagedependent fashion. Cell Stem Cell 9, 345-356 (2011).

46. Luis, T. C. et al. Wnt3a deficiency irreversibly impairs hematopoietic stem cel self-renewal and leads to defects in progenitor cell differentiation. Blood $\mathbf{1 1 3}$ 546-554 (2009) 
47. Lee, M. G. et al. Regulation of $\mathrm{Cl}-/ \mathrm{HCO}-$ exchange by cystic fibrosis transmembrane conductance regulator expressed in NIH 3 T3 and HEK 293 cells. J. Biol. Chem. 274, 3414-3421 (1999).

48. Chan, H. C. \& Sun, X. SLC26 anion exchangers in uterine epithelial cells and spermatozoa: clues from the past and hints to the future. Cell Biol. Int. 38, 1-7 (2014).

49. Kunzelmann, K. \& Mehta, A. CFTR: a hub for kinases and crosstalk of CAMP and Ca2+. FEBS J. 280, 4417-4429 (2013).

50. Melis, N. et al. Revisiting CFTR inhibition: a comparative study of CFTRinh -172 and GlyH-101 inhibitors. Br. J. Pharmacol. 171, 3716-3727 (2014).

51. Estell, $\mathrm{K}$. et al. Plasma membrane CFTR regulates RANTES expression via its Cterminal PDZ-interacting motif. Mol. Cell. Biol. 23, 594-606 (2003).

52. Reyon, D. et al. FLASH assembly of TALENs for high-throughput genome editing. Nat. Biotechnol. 30, 460-465 (2012).

53. Thisse, C. \& Thisse, B. High-resolution in situ hybridization to whole-mount zebrafish embryos. Nat. Protoc. 3, 59-69 (2008).
54. Sun, $H$. et al. Zili inhibits transforming growth factor-beta signaling by interacting with Smad4. J. Biol. Chem. 285, 4243-4250 (2010).

55. Jia, S. et al. Protein phosphatase 4 cooperates with Smads to promote BMP signaling in dorsoventral patterning of zebrafish embryos. Dev. Cell 22 1065-1078 (2012).

56. Brend, T. \& Holley, S. A. Zebrafish whole mount high-resolution double fluorescent in situ hybridization. J. Vis. Exp. 25, e1229, doi:10.3791/1229 (2009).

57. Gee HY, Noh SH, Tang BL, Kim KH, Lee MG. Rescue of DeltaF508-CFTR trafficking via a GRASP-dependent unconventional secretion pathway. Cell. 146 746-760 (2011).

58. J. B. Wallingford, R. Habas, (2005) The developmental biology of Dishevelled: an enigmatic protein governingcell fate and cell polarity. Development. 132, 4421-4436 (2005) 